\title{
Bistability and noise-enhanced velocity of rolling motion
}

\author{
Thorsten Pöschel, ${ }^{1}$ Nikolai V. Brilliantov, ${ }^{2}$ and Alexei Zaikin ${ }^{1}$ \\ ${ }^{1}$ Institut für Biochemie, Charité, Monbijoustraße 2, D-10117 Berlin, Germany \\ ${ }^{2}$ Moscow State University, Physics Department, Moscow 119899, Russia
}

(Dated: November 18, 2018)

\begin{abstract}
We investigate the motion of a hard cylinder rolling down a soft inclined plane. The cylinder is subjected to a viscous drag force and stochastic fluctuations due to the surrounding medium. In a wide range of parameters we observe bistability of the rolling velocity. In dependence on the parameters, increasing noise level may lead to increasing or decreasing average velocity of the cylinder. The approximative analytical theory agrees with numerical results.
\end{abstract}

PACS numbers: $46.30 . \mathrm{Pa}, 62.40 .+\mathrm{i}, 81.40 . \mathrm{Pq}$

Since 1785 when Vince described systematic experiments to determine the nature of friction laws [1] the effect of rolling friction has been investigated by many scientists according to its great importance in engineering and natural sciences (e.g. 2] and many references therein).

In this letter we study the rolling motion of a hard cylinder on an inclined soft plane. Experimentally it was observed that the rolling friction coefficient depends nonmonotonously on the velocity [3]: For small velocities the rolling friction force increases with increasing velocity, while for fast motion it decays as the velocity grows. Furthermore, the cylinder is subjected to the drag force of the surrounding medium, e.g. air, and unavoidably present fluctuations. We will show that this system reveals non-trivial dynamics, i.e., bistability of the cylinder's velocity and noise controlled average rolling velocity.

Model. We consider a cylinder of radius $R$, mass $M$ and moment of inertia $I$ which rolls at velocity $v$ on a plane [4]. The cylinder is subjected to an external driving force $F_{\text {ex }}=M g \sin \alpha$ due to the plane's inclination by the angle $\alpha$. The rolling friction force $F_{R}$ and the viscous drag force $F_{D}$ due to the surrounding air counteract this motion. We also assume that the tangential force acting between the cylinder and the surface at the contact area is strong enough to keep it from sliding. For certain materials it has been shown that surface effects such as adhesion may have significant influence on rolling friction (e.g. 5]). On the other hand, for viscoelastic materials it was reported that rolling friction is due very little to surface interactions, i.e., the major part is due to deformation losses within the bulk of the material 6]. Here we assume that surface effects may be neglected.

Hence, Newton's equation for the cylinder reads

$$
(M+I / R) \dot{v}=-F_{D}(v)-F_{R}(v)+F_{\mathrm{ex}}+\zeta(t) .
$$

The stochastic force $\zeta(t)$ describing fluctuations in the media is modeled by Gaussian white noise of zero average, $\langle\zeta(t)\rangle=0$, and intensity $\sigma:\left\langle\zeta(t) \zeta\left(t^{\prime}\right)\right\rangle=\sigma^{2} \delta\left(t-t^{\prime}\right)$.

For the rolling friction force $F_{R}$ we adopt the model developed in [7], where it is assumed that the plane is

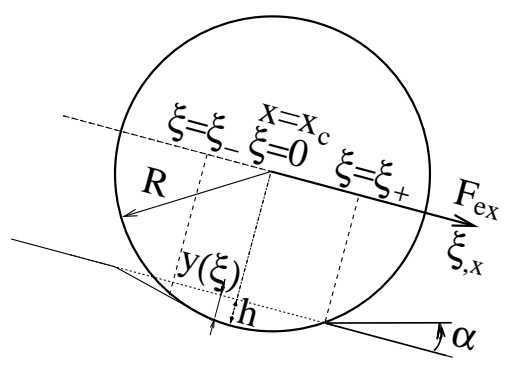

FIG. 1: A rigid cylinder of radius $R$ rolls down an inclined plane. The cylinder contacts the surface of the deformed plane in the region $\left(\xi_{-}, \xi_{+}\right)$which moves along with the cylinder.

composed of springs of elasticity $k$, mass $m$ and damping constant $\gamma$ to model elastic, inertial and viscous properties of the surface material. The rolling friction originates due to the dissipative and inertial resistance of the springs, which are compressed and subsequently decompressed when the cylinder rolls on the surface. As an important property this simple model leads to a nonmonotonous rolling friction as a function of the velocity [7] as observed in experiments [3]. Let us briefly sketch the main results of the model which will be used below. The rolling friction force is given by $[4]$

$$
F_{R}=-\frac{1}{R} \int_{\xi_{-}}^{\xi_{+}} d \xi \xi f(\xi)+m v^{2} \frac{2 h}{R},
$$

where $\xi$ is the coordinate in the comoving frame (Fig. 1), $f(\xi)$ is the corresponding force density exerted from the compressed material on the cylinder,

$$
f(\xi)=\frac{k}{2 R} \xi^{2}-\frac{\gamma v}{R} \xi+\frac{m v^{2}}{R}-h k,
$$

and $h(v)$ is the deformation. The force $f(\xi)$ acts in the contact contact area $\xi_{-}<\xi<\xi_{+}$, where the front boundary

$$
\xi_{+}=\sqrt{2 R h}
$$

follows from geometry, while for the rear boundary the 
condition $f\left(\xi_{-}\right)=0$ yields

$$
\xi_{-}=\gamma v / k-\sqrt{2 h R+\left(\gamma^{2} / k^{2}-2 m / k\right) v^{2}} .
$$

The deformation may be found from the balance of forces normal to the plane,

$$
M g \cos \alpha=-\int_{\xi_{-}}^{\xi_{+}} f(\xi) d \xi+\frac{m v^{2}}{R} \sqrt{2 h R} .
$$

The first term in Eq. (2) equals, up to the factor $1 / R$, the total torque exerted on the body due to its interaction with the deformed surface $(\xi f(\xi)$ is the torque density), while the second term in Eq. (2) as well as in Eq. (6) accounts for the surface-cylinder interaction at the infinitesimal region at $\xi_{+}$where the acceleration diverges as the cylinder rolls over (for a detailed discussion of this peculiarity see 7]). In this approach it is also assumed that the rolling body does not induce surface waves.

The viscous drag force due to the surrounding air is given by

$$
F_{D}=-A v-B v^{2}
$$

where the (positive) coefficients $A$ and $B$ depend on the Reynolds number Re, which characterizes the motion of the body. These coefficients may be estimated from hydrodynamic theory [8].

For cylinder velocity $\sim 1-10 \mathrm{~m} / \mathrm{s}$, radius $\sim 0.1-1 \mathrm{~m}$ and kinematic viscosity of the air $\nu=0.15 \cdot 10^{-4} \mathrm{~m}^{2} / s$ the Reynolds number is $\operatorname{Re} \sim 10^{4}-10^{5}$, which corresponds to the drag force $F_{D}=\frac{1}{2} c \rho v^{2} S$ with the constant $c \approx 0.5$ (here $\rho$ is the air density, $S$ - is the body cross-section) [8]. This yields the estimate for the constant $B \sim(0.01-1)$ $\mathrm{kg} / \mathrm{m}^{2}$.

For very small velocities, i.e. for $\operatorname{Re} \ll 1$, the drag force is approximately proportional to the velocity. Lamb's relation for a cylinder $\left(F_{D}=4 \pi \nu \rho v / b\right.$ with $b=\log [3.70 \nu /(\operatorname{Re} v)], \operatorname{Re} v / \nu \ll 1$ [8] ) yields the estimate for the linear constant, $A<2 \cdot 10^{-4} \mathrm{~kg} / \mathrm{s} / \mathrm{m}$. Thus, for the parameters addressed here we can neglect the linear drag force, i.e. $A=0$. We studied the above model analytically and numerically and observed the overmentioned effects of bistability and noise controlled velocity for a wide range of parameters. Here we report the results for a cylinder of mass per unit length $M=100$ $\mathrm{kg} / \mathrm{m}$ and radius $R=0.5 \mathrm{~m}$. The surface material parameters correspond to soft rubber [9]: $m=100 \mathrm{~kg} / \mathrm{m}^{2}$, $k=10^{6}-10^{7} \mathrm{~kg} / \mathrm{m}^{2} / \mathrm{s}^{2}, \gamma=10^{4}-10^{5} \mathrm{~kg} / \mathrm{m}^{2} / \mathrm{s}$ (all parameters per unit length).

Analytical approximation. As shown below for the given set of parameters the effects of interest, namely bistability of the rolling velocity and noise controlled velocity, occur at rather large values of the velocity. Therefore, as a simplification we assume that the material does not recover immediately after the cylinder has passed by, but with a delay, when the cylinder has already left. The
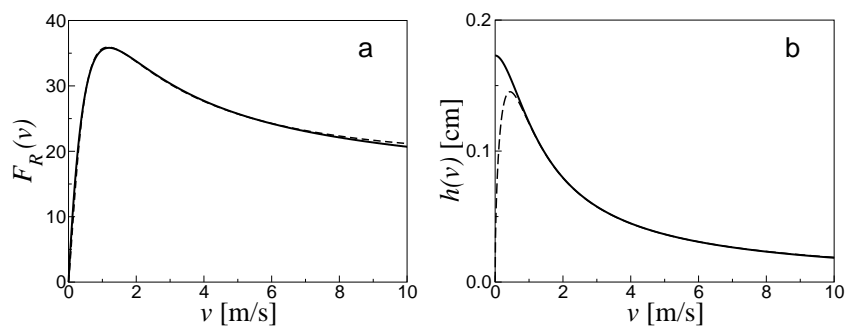

FIG. 2: a) Rolling friction force $F_{R}$ and b) deformation $h(v)$ over the velocity $v$. The full lines show the numerical solution of Eqs. 456) while the dashed lines show the approximative theory, Eqs. (8) and (9), respectively. Parameters: $m=100 \mathrm{~kg} / \mathrm{m}^{2}, k=10^{7} \mathrm{~kg} / \mathrm{m}^{2} / \mathrm{s}^{2}, \gamma=5 \times 10^{5} \mathrm{~kg} / \mathrm{m}^{2} / \mathrm{s}$, $B=0.2 \mathrm{~kg} / \mathrm{m}^{2}$.
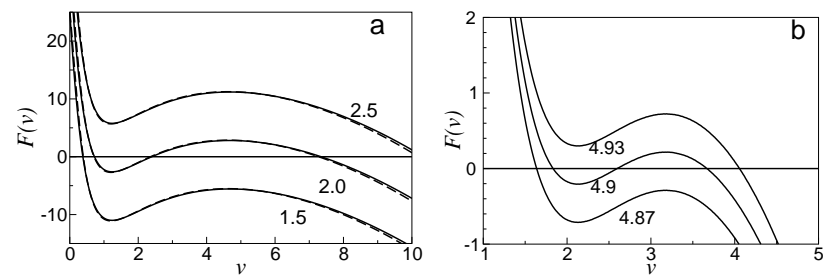

FIG. 3: Total force $F(v)$ for different angle of inclination (the number attached to the curves is the inclination in degrees). Parameters: (a) as given in Fig. 2] (b) same, except for $\gamma=$ $0.8 \times 10^{5} \mathrm{~kg} / \mathrm{m}^{2} / \mathrm{s}, k=10^{6} \mathrm{~kg} / \mathrm{m}^{2} / \mathrm{s}^{2}, B=0.65 \mathrm{~kg} / \mathrm{m}^{2}$. Full lines: numerical solution, dashed lines: approximative theory.

according approximation $\xi_{-}=0$ means that the rear part of the material does not recover on the time scale of the cylinder motion $\sim R / v$. A similar approximation was investigated in [10]. The balance equation (6) reduces then to $M g \cos \alpha=\gamma v h+\frac{2}{3} \sqrt{2 R} k h^{3 / 2}$, which yields with the condition of large damping, $v \gamma \gg k \sqrt{2 R h}$, the velocity dependent deformation

$$
h(v) \approx \frac{M g \cos \alpha}{\gamma v+\frac{2}{3} k \sqrt{\frac{2 R M g \cos \alpha}{\gamma v}}} .
$$

With the same arguments from Eq. (2) we obtain the rolling friction force

$$
F_{R}=\frac{k h^{2}}{2}+\frac{m v^{2} h}{R}+\frac{2 \gamma v h}{3 R} \sqrt{2 R h} .
$$

Bistability. Solving Eqs. (456) we obtain the deformation $h(v)$ as a decaying function of the velocity $v$ (Fig. 2b), i.e., the faster the cylinder moves, the less the surface is compressed. Using the obtained deformation $h(v)$, the corresponding rolling friction force may be calculated (Fig. 2a). $F_{R}$ depends non-monotonously on the velocity, first it increases while at larger velocity it decreases. The non-monotonous behavior of $F_{R}(v)$ results from the interplay between the amount of deformed 

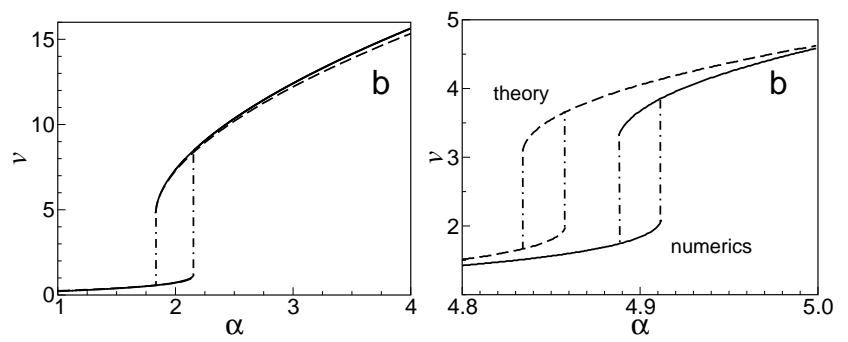

FIG. 4: Bifurcation diagram for stable velocity states (full line: numerical solution, dashed line: analytical approximation). (a) corresponds to Fig. 2 and Fig. 37, (b) corresponds to Fig. [3 p. In both cases the agreement between numerics and theory is within few percent.

material and the deformation rate: For small velocity, $F_{R}(v)$ increases since the surface material is compressed at increasing rate with growing $v$ while at higher rolling velocity the amount of deformed material decreases as $h(v)$ decays. Consequently for fast motion the rolling friction force decays with increasing velocity.

Given the non-monotonous rolling friction force, the total force acting on the cylinder, $\mathcal{F}(v)=F_{D}+F_{R}+F_{\text {ex }}$, is represented for realistic parameters by a $S$-shaped curve, implying bistability of the velocity for a certain range of parameters. Indeed, the steady state condition, $\dot{v}=0$, or $\mathcal{F}(v)=0$ may be fulfilled either for only one velocity (top and bottom curves in Figs. [3or for three different velocities (middle curves). The former case implies a unique stationary velocity, while the later case allows for three velocities. Only two of them, the smallest and the largest, correspond to stable motion $(\partial \mathcal{F} / \partial v<0)$. By varying the parameter of the drag force $B$, or by modifying the driving force $M g \sin \alpha$ the system may transit from one stable solution to the other. Figure 4 shows the bifurcation diagram.

Starting from an inclination angle that corresponds to a monostable velocity, the variation of $\alpha$ causes the appearance of the bistable regime, change of the symmetry of the potential $U(v)$ (Fig. 5) and the transition to the other monostable regime.

As shown in Figs. 24 the analytical theory (dashed curves) agrees well with the numerical results if the damping parameter $\gamma$ is large. The deviation in Fig. 4 appears large due to the very detailed resolution of the horizontal axes, in relative units the deviation is few percent.

Noise-induced jumps. Up to now we did not consider noise, which is always present in realistic systems. If the system has only one stable velocity, the addition of noise does not change the motion of the cylinder qualitatively. In this case the velocity fluctuates around the average value given by the steady state condition $\mathcal{F}(v)=0$. Figure 6] (top left and right) shows the velocity of the cylinder as obtained from a numerical integration of the

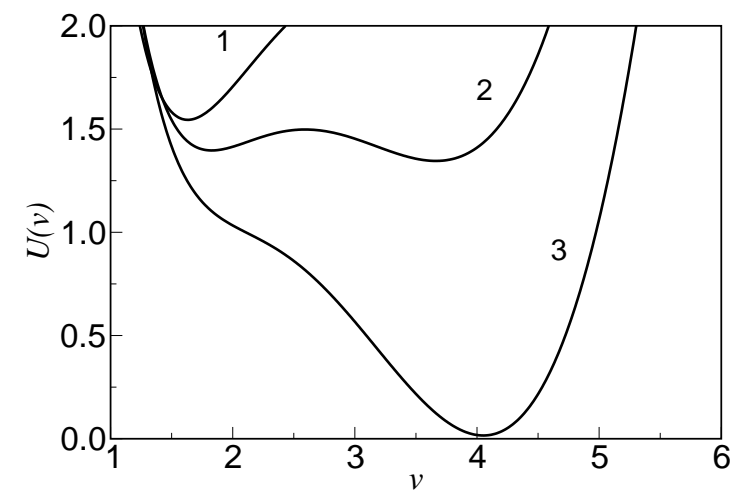

FIG. 5: Force potential $U(v)(F(v)=-d U / d v)$ over the velocity $v$ (parameters as given in Fig. 2) for angle of inclination $1^{\circ}, 2^{\circ}$ and $3^{\circ}$.
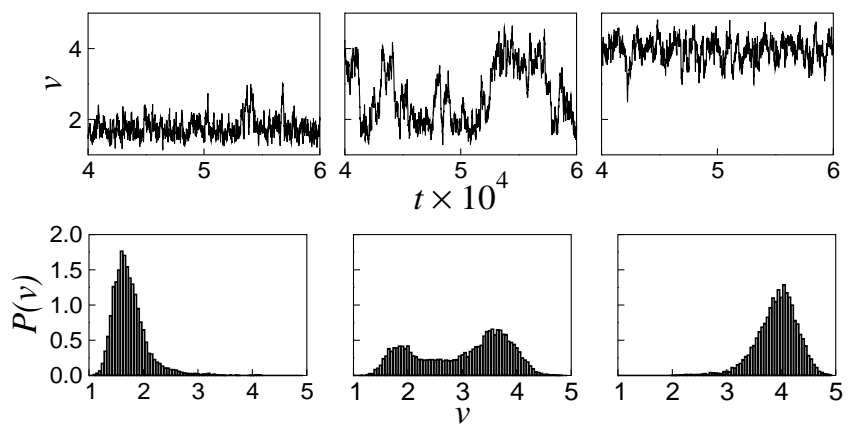

FIG. 6: Top: velocity of the cylinder subjected to Gaussian white noise of intensity $\sigma^{2}=50 \mathrm{~kg}^{2} / \mathrm{s}^{3}$ as obtained from the numerical integration of Eq. (1). Bottom: the corresponding velocity distributions of the cylinder. From left to right: $\alpha=$ $4.87^{\circ}, 4.9^{\circ}, 4.93^{\circ}$, all other parameters are the same as in Fig. $3 \mathrm{~b}$.

stochastic equation (11). The corresponding velocity distribution reveals a single peak (Fig. [6] bottom left and right).

For parameters which correspond to bistable velocity the presence of the noise changes the system behavior qualitatively. Instead of moving with a fixed velocity there are stochastic jumps between the meta-stable velocities. Figure 6 (top, middle) illustrates this regime. Consequently, the velocity distribution reveals two well separated peaks (Fig. [6] bottom, middle).

Noise-enhanced velocity. Under certain conditions due to the nonlinear dependence of the rolling friction on the velocity the average cylinder velocity may increase with increasing noise level. Since $v$ increases at the same driving force $F_{\mathrm{ex}}$, one effectively has increased mobility $\mu=v / F$. This surprising effect is shown in Fig. 7

This phenomenon may be understood analyzing the dynamics of the system with respect to the force potential $U(v)$. In the absence of noise the velocity remains in its (meta-)stable state, where $d U / d v=-\mathcal{F}(v)=0$, i.e., at the minimum of the potential $U(v)$ (Fig. 5). Subjected to 

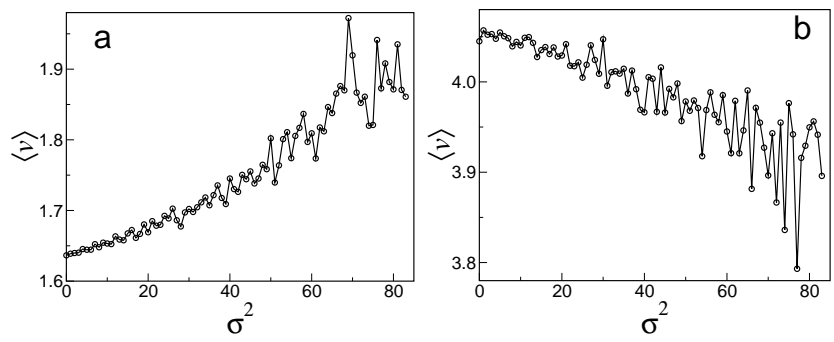

FIG. 7: Mean velocity of the cylinder over the noise intensity. (a) $\alpha=4.87^{\circ}$, (b) $\alpha=4.93^{\circ}$. The parameters of the system are given in Fig. 3 p.

the noise, the velocity of the cylinder fluctuates around this minimum. If the potential $U(v)$ has two minima, the velocity jumps between both potential wells. If one potential well of $U(v)$ is narrow while the other is wide, the average velocity will be shifted towards the wider well. This shift increases with increasing transition rate between the wells. If the potential well is wider for the larger stable velocity than for the smaller one, the average velocity, thus, increases with increasing noise level (Fig. 77). In the opposite case, large noise impedes rolling (Fig. [7b).

Summary. We investigated the rolling motion of a hard cylinder on an viscoelastic plane in the presence of a surrounding medium. The motion of the cylinder is driven by an external force due to the inclination of the plane. The rolling friction force and the viscous drag force due to the surrounding medium counteract its motion. For certain realistic parameters the stationary velocity of the cylinder is bistable. The numerical results agree well with the predictions of a simplified analytical theory in the limit of large damping.

In the presence of noise as it is unavoidable in any realistic experiment, noise-induced transitions between the meta-stable velocities are observed. Depending on the system parameters, increasing noise level may accelerate or decelerate the rolling motion.

The described effects may be important for technical systems where the addition of noise may lead to an effective increase of the mobility of a rolling body driven by an external force.
[1] S. Vince, Phil. Trans. Roy. Soc. London 75, 165 (1785).

[2] H. Czichos, Tribology Elsevier (Amsterdam, 1978); F. T. Barwell, Bearing Systems: Principles and Practice, Oxford Univ. Press (Oxford, 1979); A. Z. Szeri, TribologyFriction, Lubrication, and Wear, Hemisphere (Washington, 1980); D. Dowson, C. M. Taylor, T. H. C. Childs, M. Godet, and G. Dalmaz (Eds.), Dissipative Processes in Tribology, Elsevier (Amsterdam, 1994); G. P. Shpenkov, Friction Surface Phenomena, Elsevier (Amsterdam, 1995); E. Rabinowicz, Friction and Waer of Materials, Wiley (New York, 1965);

[3] B. J. Olson, S. W. Shaw, and G. Stépán, Vehicle System Dynamics 40, 377 (2003); J. Yi, L. Alvarez, and R. Horowitz, IEEE Transactions on Control Syst. Techn. 10, 381 (2002); F. Gustafsson, Automatica 33, 1087 (1997); L. R. Ray, ibid 33, 1819 (1997); E. Bakker, L. Nyborg, and H. Pacejka, Soc. Automotive Eng. Paper \# 870421 (1987); M. Burckhardt, Fahrwerktechnik: RadschlupfRegelsysteme, Vogel-Verlag (Würtzburg, 1993); K. Yi and T. Jeong, JSME Int. J. 41, 116 (1998).

[4] Quantities such as $M, F_{R}, F_{\text {ex }}, I$, describing the cylinder, are given as per unit length of the cylinder. Therefore, e.g., $M$ is measured in $\mathrm{kg} / \mathrm{m}$. Consequently, the units of the quantities, describing the material of the inclined plane are $[k]=\mathrm{kg} / \mathrm{m}^{2} / \mathrm{s}^{2},[\gamma]=\mathrm{kg} / \mathrm{m}^{2} / \mathrm{s}$, and $[m]=\mathrm{kg} / \mathrm{m}^{2}$.

[5] M. Barquins, D. Maugis, J. Blouet, and R. Courtel, Wear 51, 375 (1978); B. V. Deryaguin and Y. P. Toporov, Progr. in Surface Sci. 45, 317 (1994); K. Kendall, Wear 33, 351 (1975); K. N. G. Fuller and A. D. Roberts, J. Phys. D 14, 221 (1981); A. D. Roberts and A. G. Thomas, Wear 33, 45 (1975).

[6] D. Tabor, Proc. Roy. Soc. A 229, 198 (1955); 43, 1055 (1952); D. Atack and D. Tabor, Proc. Roy. Soc. A 246, 539 (1958); R. C. Drutowski, In R. Davies (Ed.), Proc. Symp. Friction and Wear, Elsevier (Amsterdam, 1959), p. 17.

[7] T. Pöschel, T. Schwager, and N. V. Brilliantov, Eur. Phys. J B 10, 169 (1999).

[8] L. D. Landau and E. M. Lifshitz, Hydrodynamics, Pergamon (New York, 1986).

[9] Landolt-Börnstein. New Series, V/1b, Springer (Berlin, 1982).

[10] J. A. Greenwood, H. Minshall, and D. Tabor, Proc. Roy. Soc. A 259, 480 (1961). 